\title{
A test of the predicted relation describing the change in response rate with the number of intervals on an FI schedule
}

\author{
ERNEST DZENDOLET \\ University of Massachusetts, Amherst, Massachusetts
}

\begin{abstract}
Two experiments, one with rats and the other with pigeons, supported the prediction that the logarithm of the rate of responding in the last fifth of a fixed interval is linearly related to the logarithm of the number of intervals to which the organism is exposed. This prediction was derived with the use of logical positivism from Hull's law on delay of reinforcement. The support of this relation strengthens the possible role that deterministic equations can play in learning theory.
\end{abstract}

In an earlier paper (Dzendolet, 1984), it was predicted that the logarithm of an organism's rate of responding, under a fixed-interval (FI) schedule, would be linearly related to the logarithm of the number of reinforcements: for example,

$$
\log R=(b-a \sqrt{F I}) \log T+\log F,
$$

in which $R$ is the rate of responding, $T$ is the number of reinforcements or trials, and FI is the value of the fixed interval. The quantities $\mathrm{a}, \mathrm{b}$, and $\mathrm{F}$ are constants. This equation was derived from Hull's (1951) law concerning the delay of reinforcement.

The purpose of the current paper is to report the results of a test of Equation 1. Two experiments were carried out, one with rats and the other with pigeons.

\section{EXPERIMENT 1}

\section{Method}

Subjects. Two naive male albino rats, 150 and 180 days old, from Gofmoor Farms, Westboro, Massachusetts, were reduced to approximately $80 \%$ of their free-feeding body weights and were kept at that percentage for the duration of the experiment.

Apparatus. Two Gerbrands operant conditioning chambers, with dipper feeders and response bars, were used. The chambers were placed in a soundproof box, and an 87-dB SPL white noise was always present as background masking. A $32 \% \mathrm{w} / \mathrm{w}$ commercial sucrose solution was prepared and then kept under refrigeration to prevent spoilage; the solution was presented, cold, in the 0.1-cc dipper cup in order to maintain responding. Further details of the equipment are given in DeCosta and Ayres (1971).

Procedure. After 10 days of restricted feeding, the rats were taught over 2 days to lick the sucrose solution from the dipper cup. Over the next 2 days, they were taught to barpress. For each barpress, they had access to the dipper cup for approximately $4 \mathrm{sec}$. In the following 2 days, the rats were placed on a continuous reinforcement schedule for about 100 reinforcement presentations each day. The next day, they were placed on an FI 18-sec schedule, for a total of 30 reinforcements. The

The author's mailing address is: Department of Psychology, University of Massachusetts, Amherst, MA 01003. following day they were placed on an FI 30-sec schedule, again for 30 reinforcements. The next day they were changed to an FI 60-sec schedule, for the same number of presentations. The subjects continued on this same schedule for a total of 18 daily sessions. On the 19th day, the schedule was changed to FI $30 \mathrm{sec}$, and the rats continued under this condition for another 15 days, at which time the experiment was ended.

In recording the barpress data, each interval between reinforcements was divided into fifths, and the number of responses in each fifth was cumulated in each daily session over the 30 reinforcements. The total number of daily responses in the last fifth of the interval was the basic datum in this experiment. The rationale for the use of the last fifth was given earlier (Dzendolet, 1984). This number of responses was converted to a rate (responses/second) for both the FI 60-sec and FI 30-sec schedules. Finally, a 3-day arithmetic mean was calculated for the responses under both schedules.

\section{Results}

The 3-day mean rates of responding of each rat were plotted against sessions or days, on log-log coordinates, as shown in Figures 1 and 2. Least-squares fits were cal-

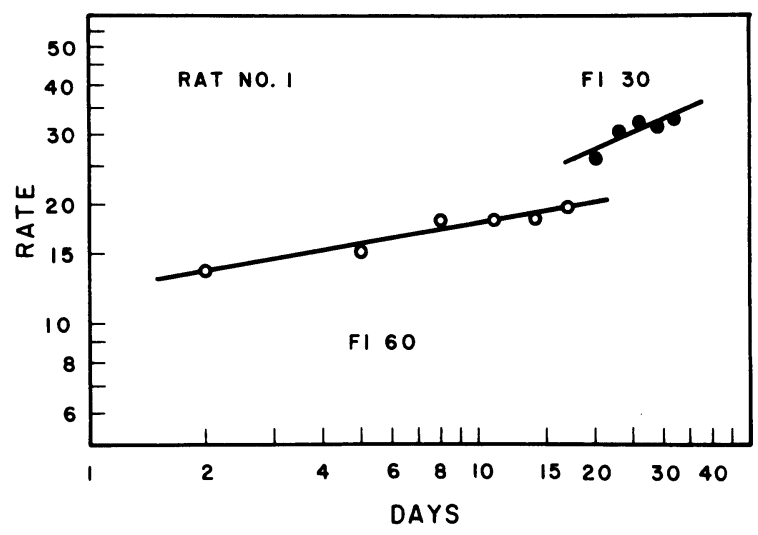

Figure 1. Rate of barpressing in the last fifth of each FI interval, for Rat 1 , as a function of days. The solid lines are least-squares fits to the indicated data points. Further details are given in the text for the slope values and coefficients of determination, for these curves, and for Figures 2-4. 


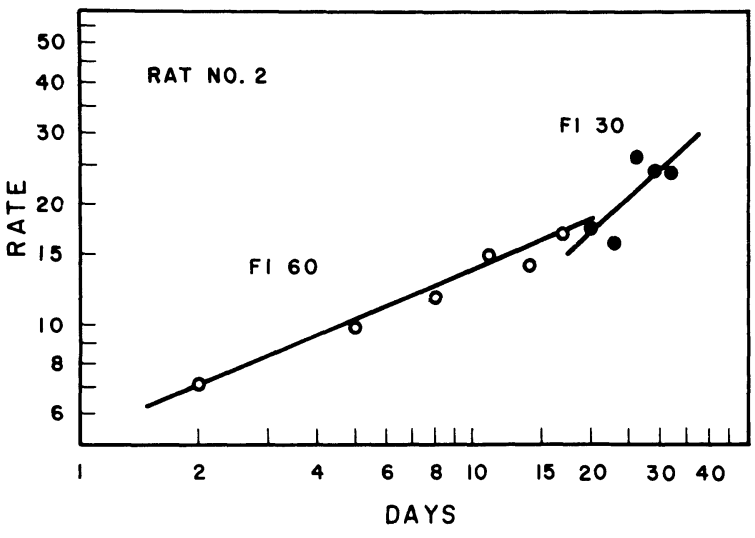

Figure 2. Data similar to Figure 1, but for Rat 2. There is greater variability in the responding on FI $30 \mathrm{sec}$.

culated separately for the FI 60-sec and the FI 30-sec data, and are indicated by the solid lines.

For Rat 1, and the FI 60-sec schedule, the slope was +0.17 , and the coefficient of determination, $\mathrm{r}^{2}$, was 0.90 . For the FI 30-sec schedule, the slope was +0.43 , and $\mathrm{r}^{2}$ was 0.75 . It is clear that the latter is not as good a fit as the former, but a clear discontinuity between the responses in the two schedules seems obvious.

For Rat 2, and the FI 60-sec schedule, the slope was +0.41 , and $\mathrm{r}^{2}$ was 0.96 . For the FI 30 -sec schedule, the slope was +0.90 , and $\mathrm{r}^{2}$ was 0.58 , a much poorer fit than the FI 30 -sec fit for Rat 1.

\section{Discussion}

For both rats, the prediction of Equation 1 was supported; that is, the data were described by a linear function. Furthermore, when the value of the FI changed, the slope of the function changed, again, a finding in consonance with the prediction of Equation 1.

The constants $\mathrm{a}$ and $\mathrm{b}$ of Equation 1 can be calculated because we have two equations-the values of the two slopes at the two FI schedules and the two unknowns. When these two equations were solved simultaneously for Rat 1 , a was found to be 0.1140 and b, to be 1.053 . From these constants for this rat, an FI value can be calculated, at which the slope of this rat's function would be zero; that is, the rate over days would remain constant. Using Equation 1 and setting the slope at zero, and with the $a$ and $b$ values determined above, the FI value for this rat would be $85.3 \mathrm{sec}$.

In the same way, the values for $\mathrm{a}$ and $\mathrm{b}$ of Rat 2 were determined. They were: $a=0.2160$ and $b=2.0805$. Similarly, the FI value for a zero slope can be calculated for this rat: $92.8 \mathrm{sec}$.

The two zero-slope values determined above appear to be reasonably close to one another, the larger being $8.8 \%$ greater than the smaller. This suggests that the approximate value of $90 \mathrm{sec}$ might be considered as a characteristic for this strain of rats. That is, any FI value below $90 \mathrm{sec}$ would produce a positive slope, and any value above $90 \mathrm{sec}$, a negative slope.

\section{EXPERIMENT $2^{2}$}

The purpose of this experiment was to see if the findings of Experiment 1 would be confirmed in a different species.

\section{Method}

Subjects. Two White Carneaux pigeons were maintained at approximately $75 \%$ of their free-feeding weights throughout the experiment.
They had been used earlier in a study that had investigated problems in stimulus control; in that study, they had been trained to keypeck a variable-interval reinforcement schedule.

Apparatus. This was a pigeon operant chamber (Lehigh Valley Electronics), enclosed by a sound-attenuating wooden hull. Only the righthand response key of the chamber was operative, and it was transilluminated by light passing through a green filter (Kodak Wratten filter No. 99). White noise was also delivered during the experimental session at $85 \mathrm{~dB}$ SPL by means of a speaker mounted on the front wall of the chamber. Reinforcement consisted of a 4-sec access to a grain hopper through an aperture located to the left of the operative response key. Further details are given in Couch (1975).

Procedure. Both pigeons were placed on an FI 15-sec schedule and then on an FI 30 -sec schedule for 4 days each, as preliminary training. The experiment proper began on the 9th day, when the pigeons were shifted to an FI 60-sec schedule. Each pigeon was given one experimental session per day, with 30 reinforcements defining the daily session. After 15 days on the FI 60 -sec schedule, the pigeons were shifted to FI $30 \mathrm{sec}$, and the experiment was continued for an additional 24 days. It was assumed that if the linearity on FI $60 \mathrm{sec}$ that had been shown by the rats were to occur with the pigeon, it would show clearly in 15 days. In addition, the amount of time on FI $30 \mathrm{sec}$ was extended, as mentioned earlier, for a further 7 days, in order to see what effect this would have on the linearity of the function.

As with the rats, each interval between reinforcements was divided into fifths, and the number of responses in each fifth was cumulated in each daily session over the 30 reinforcements. As before, the total number of daily responses in the last fifth of the interval was the basic datum, and this number was converted to a rate (responses/second). Using these daily rates, 3-day arithmetic means were calculated for the responding under both schedules.

\section{Results}

The 3-day means were plotted against days, on log-log coordinates; the results are shown in Figures 3 and 4. Least-squares fits were calculated separately for the two schedules, and are indicated by the solid lines through the data points in Figures 3 and 4. In the case of Pigeon 19, all eight of the points on the FI 30-sec schedule appear to define an inverted- $V$ curve. Because none of the other data in this experiment showed such an effect, the first point in this schedule was not used in the least-squares fit; only the remaining seven means were used.

For Pigeon 18 on the FI 60 -sec schedule, the slope was -0.038 , and the coefficient of determination, $r^{2}$, was

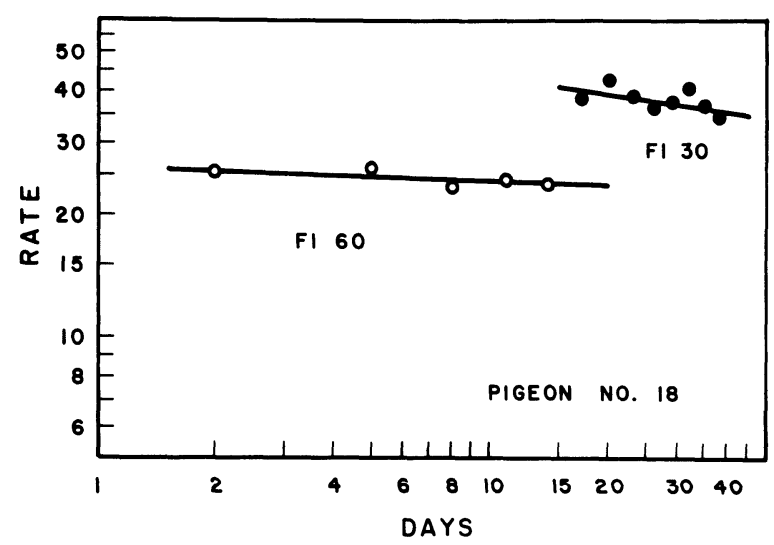

Figure 3. Data similar to those in Figures 1 and 2, but for Pigeon 18. The number of days over which the bird was tested on FI $30 \mathrm{sec}$ was increased, and a periodicity in response rate appears to be present. 


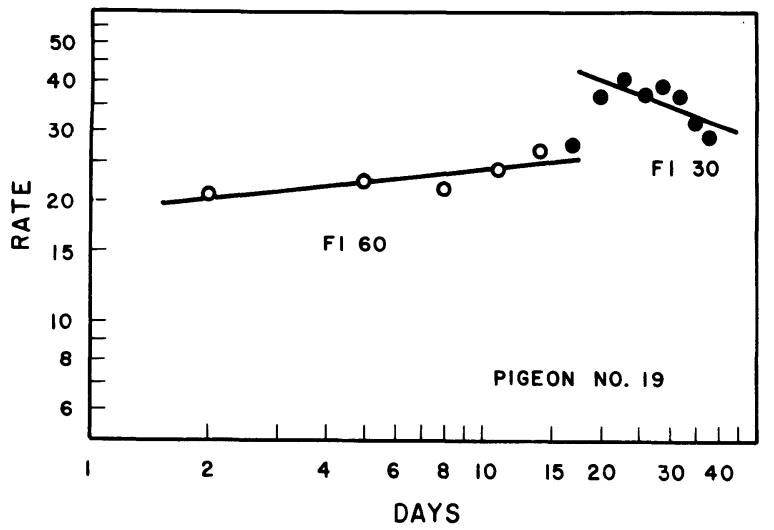

Figure 4. Data for Pigeon 19. The transition to FI $30 \mathrm{sec}$ was not as quick as that with Pigeon 18. A periodicity still seems to be present with FI 30 sec.

0.46 . For the FI 30 -sec schedule, the slope was -0.14 , with an $\mathrm{r}^{2}$ of 0.34 . In this pigeon, a clear discontinuity was present between the rates of responding on the two schedules.

The data from Pigeon 19 showed a slope of +0.10 , with an $\mathrm{r}^{2}$ of 0.62 for the FI 60 -sec schedule. For the FI 30 sec schedule, without the first mean, the slope was -0.38 , with an $\mathrm{r}^{2}$ of 0.54 .

\section{Discussion}

The prediction of linearity by Equation 1 was supported by the data from these pigeons. As with the rats, a change in the value of the FI also changed the slope of the resulting function. This transition, however, was slower with Pigeon 19 than with Pigeon 18. In that respect, Pigeon 18's performance seems to have resembled that of Rat 2, as shown in Figure 2.

The constants $\mathrm{a}$ and $\mathrm{b}$ can also be calculated for the pigeons as they were for the rats. For Pigeon 18, a becomes -0.0433 , and b, -0.3733 . The FI value for a zero slope proves to be $74.3 \mathrm{sec}$. For Pigeon 19, $a$ is -0.2127 , and $b,-1.5437$. The FI value for this pigeon for a zero slope is $52.7 \mathrm{sec}$, and the arithmetic mean of the two is $63.5 \mathrm{sec}$. The percentage difference between the two zero-slope values is $41.0 \%$, a much larger percentage than that found for the rats.

\section{GENERAL DISCUSSION}

It seems clear that the data from these two experiments are consistent in supporting the prediction of Equation 1 and that, for the individual organism, the slope of the linear function is related only to the FI value.

The calculation of the zero-slope FI value shows an interesting difference between the rats and the pigeons. In the rat, an FI value less than the zero-slope FI will produce a slope that is positive. In the pigeon, however, a value less than the zero-slope value will produce one that is negative. Similarly, an FI value greater than the zero-slope value will, in the rat, produce a negative slope, whereas, in the pigeon, it will produce a positive slope.

The fact that the response rate can decrease over time, at least with some FI values, is not an unexpected finding. This merely means that the number of responses in the last fifth of the FI interval decreases. The possibility of such a decrease had been suggested earlier (Ferster \& Skinner, 1957, p. 266). The argument given was that if an organism were to possess an accurate internal clock with which to time the duration of the interval, only a single response would be needed to obtain the reinforcement. On this basis, the data from the current experiments can be used to argue that rats are better able to time longer intervals than they are shorter ones, because the slope of the log rate versus log Days curve decreases with longer FI values. The opposite is true of pigeons; that is, they are able to time shorter FI values better than they are longer ones.

An explanation of this apparent difference between the rat and the pigeon, following logical positivistic lines, is straightforward. The constants $\mathrm{a}$ and $\mathrm{b}$ in the slope expression, $\mathrm{b}-\mathrm{a} \sqrt{\mathrm{FI}}$, are similar in the rat and the pigeon to the extent that $b$ is greater than $a$ in absolute value. However, in the rat, they are both positive, whereas, in the pigeon, they are both negative. This fact effectively causes a change in the sign of the slope expression, leading to the difference described in these two species.

Another point is the appearance of an oscillatory behavior in the rate of responding. Such an oscillation was noted by Ferster and Skinner (1957, p. 149), but was described as occurring primarily across adjacent intervals. The data of Hays (1946; as cited in Hull, 1951, pp. 41-42), on the other hand, show such oscillations as a function of days, as do the results of the current experiments. The variable in Hays's experiment was the reciprocal of jumping-stand latency. More recently, Zeiler and Davis (1978) reported the occurrence of irregular oscillations, over days, in the response rate of pigeons that were on various FI schedules. Zeiler and Davis's results are not as periodic as those in the current study, perhaps because they calculated the response rate by using all the responses in the entire interval, and not merely those in the last fifth. In addition, the 3-day mean in the current study tends to smooth the effect of daily irregularities.

The results of the two experiments described above have a bearing on the issue of stability of behavior. Whether or not the four organisms in this study exhibited stable behavior would depend on the definition of the word, stable. If regularity of behavior is equated with stability, then all of the organisms showed stability, because their data all followed a given mathematical function, that is, Equation 1. Even the presence of the oscillation under FI $30 \mathrm{sec}$ does not preclude stability, because it appears to be a known phenomenon, although its wavelength has not been determined yet. On the other hand, if stability is taken to mean an unchanging rate of responding, only Pigeon 18, under FI $60 \mathrm{sec}$, seemed to come close to this criterion. In all other cases, the organisms were either increasing or decreasing their rates as a function of days, with no apparent asymptotic value in view.

It appears that, at least with an FI schedule, a different approach to the issue of stability should be taken. Instead of using a criterion such as one of those evaluated by Killeen (1978), an experimenter who wishes to use rate as his/her measure should use the zero-slope value for his/her organism as his/her baseline, perhaps refining the initial FI choice, as necessary, after the first 9 days. Thereafter, any change in rate due to the introduction of an experimental variable could be more closely related to that variable, and any statistical treatment would be more likely to show significance if a difference were indeed present. Alternatively, in order to save time, the experimenter need not refine his/her initial choice of the FI, but could use an estimate of the rate, based on the initial function, at the time at which he/she would be measuring the rate under the experimental condition.

Finally, the results of this investigation show that simple mathematical manipulations or transformations of concepts, such as the conversion of latencies into rates, are as valid in the area of learning as they are in other sciences. It also appears that there is still a place for an island of determinism in the midst of the current ocean of probabilistic approaches to learning.

\section{REFERENCES}

Couch, J. V. (1975). Behavioral contrast and inhibitory stimulus control. Animal Learning \& Behavior, 3, 347-358.

DeCosta, M. J., \& AYRES, J. J. B. (1971). Suppression of operant vs. consummatory behavior. Journal of the Experimental Analysis of Behavior, 16, 133-142.

Dzendolet, E. (1984). A correction to Hull's law on delay of reinforcement and its extension to fixed-interval operant schedules. Bulletin of the Psychonomic Society, 22, 451-454. 
Ferster, C. B., \& SKINNER, B. F. (1957). Schedules of reinforcement. New York: Appleton-Century-Crofts.

Hull, C. L. (1951). Essentials of behavior. New Haven, CT: Yale University Press.

KILLEEN, P. R. (1978). Stability criteria. Journal of the Experimental Analysis of Behavior, 29, 17-25.

ZEILER, M. D., \& DAvis, E. R. (1978). Clustering in the output of behavior. Journal of the Experimental Analysis of Behavior, 29, 363-374.

\section{NOTES}

1. I am indebted to J. J. B. Ayres for the use of his apparatus, and to $\mathrm{R}$. Sacks for his aid with the computer program.

2. I am indebted to J. W. Donahoe for the use of his apparatus, and to $\mathrm{J}$. V. Couch for aid in its programming.

(Manuscript received for publication June 24, 1984.) 